\title{
Transactional Pre-abort Handlers in Hardware Transactional Memory
}

\author{
Sunjae Park \\ Georgia Institute of Technology \\ sunjae.park@gatech.edu
}

\author{
Christopher J. Hughes \\ Intel \\ christopher.j.hughes@intel.com
}

\author{
Milos Prvulovic \\ Georgia Institute of Technology \\ milos@cc.gatech.edu
}

\begin{abstract}
Commercially available hardware transactional memory (HTM) implementations resolve a number of potentially problematic situations by aborting a transaction, rather than add the hardware complexity needed to handle each situation more gracefully.

In this paper we propose pre-abort handlers, a general-purpose mechanism that invokes a software handler instead of aborting the transaction. The handler is executed non-transactionally while a transaction's execution is paused, allowing the handler to decide on and then perform the actions appropriate for the specific situation that caused the handler to be invoked.

We use our pre-abort handler mechanism to salvage a transaction's already-performed work in several common scenarios, and also to allow controlled nesting of non-transactional work within a transaction, without further changes to the hardware (beyond pre-abort handler support itself). In contrast, prior work on abort mitigation and on allowing non-transactional work within transactions requires dedicated hardware support for mitigation of each type of problematic situation.
\end{abstract}

\section{CCS CONCEPTS}

- Computer systems organization $\rightarrow$ Multicore architectures;

\section{KEYWORDS}

Transactional Memory,Multicore,Mutex Locks

\section{ACM Reference Format:}

Sunjae Park, Christopher J. Hughes, and Milos Prvulovic. 2018. Transactional Pre-abort Handlers in Hardware Transactional Memory. In International conference on Parallel Architectures and Compilation Techniques (PACT '18), November 1-4, 2018, Limassol, Cyprus. ACM, New York, NY, USA, 11 pages. https://doi.org/10.1145/3243176.3243186

\section{INTRODUCTION}

Transactional memory (TM) promises improved performance for critical sections through optimistic concurrency control, i.e. by optimistically executing critical sections (transactions) and detecting potential atomicity violations (data conflicts) in the process [15]. This allows non-conflicting critical sections to execute concurrently, improving performance over the traditional approach that uses

Permission to make digital or hard copies of all or part of this work for personal or classroom use is granted without fee provided that copies are not made or distributed for profit or commercial advantage and that copies bear this notice and the full citation on the first page. Copyrights for components of this work owned by others than ACM must be honored. Abstracting with credit is permitted. To copy otherwise, or republish, to post on servers or to redistribute to lists, requires prior specific permission and/or a fee. Request permissions from permissions@acm.org.

PACT '18, November 1-4, 2018, Limassol, Cyprus

(C) 2018 Association for Computing Machinery.

ACM ISBN 978-1-4503-5986-3/18/11 ..\$15.00

https://doi.org/10.1145/3243176.3243186 mutual exclusion (mutex) locks, where only one among threads protected by the same lock is allowed into the critical section even when there is no actual conflict.

On the other hand, when there is a conflict, it must be resolved to prevent atomicity from being violated. Typically this is done by aborting and retrying one (or both) of the conflicting transactions. When a transaction is aborted, the work done so far is discarded, and the thread's state is rolled back to just before the transaction started. In hardware TM (HTM) implementations, this is done automatically because a transaction that has encountered an abort-causing condition is seemingly "doomed."

However, some are salvageable. For example, in commercial HTM systems, speculative data is buffered in a private buffer (such as the private cache) until the transaction is committed $[6,17,18]$. If the speculative data does not fit within this private buffer, the transaction can no longer continue and is aborted, although there were no data conflicts. Similarly, a system call results in aborting the transaction $[6,17,18,28]$.

Commercial hardware TM systems are also best effort in that they make no forward progress guarantees. As a result, software needs to provide an alternative means of providing forward progress, called the fallback path [17]. One common implementation of the fallback path is to execute the transaction as a critical section, whose mutual exclusion is enforced by aborting all other active transactions, even though no data conflicts have been detected.

Overall, commercial HTM implementations have a number of situations that cause transactions to abort for reasons other than data conflicts. Although the speculative work done so far is still perfectly valid, the limitations of the HTM implementation may prevent these situations from being correctly handled. As a result, the work is discarded.

Irrevocable Transactions [21,29] is one solution that was proposed to avoid this type of abort. If this situation occurs, the transaction can be marked as irrevocable, or non-abortable, effectively converting the transaction into a critical section. However, this conversion must be done before the abort. OneTM does this conversion automatically in hardware [4], but at the cost of adding special-purpose functionality to the TM hardware.

Moreover, abort-mitigating actions like transaction conversion may need to be implemented in a non-speculative manner. Nontransactional operations within a hardware transaction have been explored before, either by using explicitly non-speculative load and store operations $[8,9]$, or via transaction suspend and resume operations [6]. Either way, these require additional hardware specific for this purpose as well.

Instead, it would be preferable to have a generic mechanism that can be used to support mitigating actions, and possibly other 
purposes. Thus we propose that the HTM, instead of simply aborting the transaction upon detecting an action that creates an abortcausing condition, triggers execution of a pre-abort software handler without aborting the transaction ${ }^{1}$. This handler executes outside of the speculative context, and can implement mitigating actions that allow the transaction to continue, or simply allow the transaction to be aborted, e.g. when abort is actually needed or when the appropriate mitigating action is not implemented in the handler. To provide the handler with the information necessary to decide which action to take, the type of the abort trigger and additional arguments such as relevant instruction and data addresses is passed to the handler.

We implement our approach in SESC [25], a cycle-accurate simulator, and show that several types of aborts can be avoided using pre-abort handlers. By avoiding these non-conflict aborts, we can reduce the impact of micro-architectural HTM limitations and allow programmers to focus on more algorithmic issues such as data conflicts instead of worrying about whether HTM limitations will cause their application to have disastrous TM performance. We also show additional use cases for pre-abort handlers, such as inserting non-transactional logging within transactions.

To the best of our knowledge, this is the first paper to split the abort condition detection and state rollback, and insert general purpose code (the pre-abort handler) in between the two. The rest of the paper is organized as follows: Section 2 explains in more detail how transactions are used in commercial HTM systems. Section 3 introduces pre-abort handlers, which allow transactions that encounter exceptional, abort-causing situations to take mitigating action. Section 4 describes various use cases of our handler mechanism. Section 5 discuss our evaluation results, and Section 6 goes through related work. Finally we wrap up in Section 7.

\section{BASELINE IMPLEMENTATION OF TRANSACTIONS}

In a transaction, a thread optimistically works on shared data while checking for conflicts that (may) violate the transaction's atomicity. The TM system keeps track of which data each transaction has accessed: the read set and write set. Using these sets, transactions identify any potential read-write or write-write conflicts, and abort one (or more) of the conflicting transactions when a conflict is detected.

A transaction abort involves discarding its speculative state, placing some information about the cause of the abort in a status register, and then passing control to an abort handler. This handler chooses the desired course of action, e.g. retry the transaction immediately, retry after a brief wait, or use the fallback path.

The fallback path is an alternative to retrying the transaction, and is needed because commercial HTM implementations are "besteffort," i.e. they do not in general guarantee forward progress of transactions. For example, a transaction whose working set is larger than the private storage will be aborted on every retry, so the abort handler must eventually use the fallback path for such a transaction. A typical fallback path acquires a mutex lock, the fallback lock. The

\footnotetext{
${ }^{1}$ Note that this is different from the regular abort handler, which is called after the transaction is aborted
}

thread then executes the same code as the transaction's body, but non-speculatively, as a critical section.

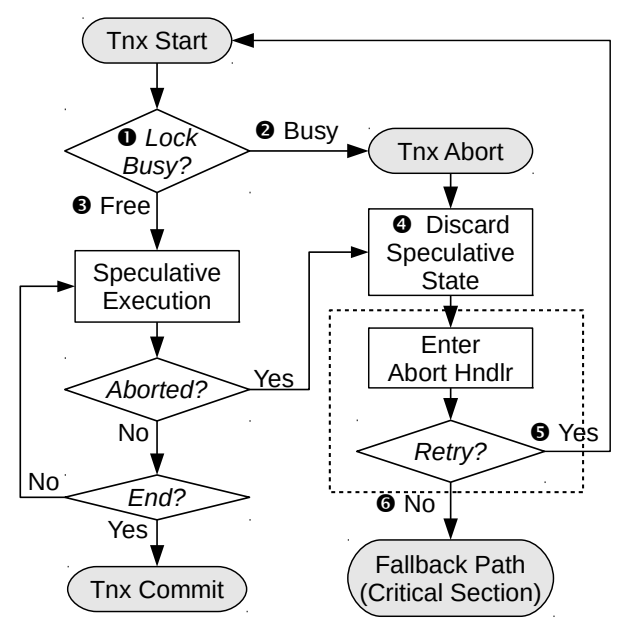

Figure 1: Life of a Transaction with the Baseline Fallback

The life a typical transaction is shown in Figure 1. First, it checks the status of the fallback lock (1). This must be done inside the transaction to eliminate the race between the check and the start of the transaction, and to add the fallback lock to the transaction's read set. This ensures that the transaction will detect a conflict (and be aborted) if another thread acquires (writes to) the lock.

A busy lock (2) means that another thread is using the fallback path, so the transaction aborts itself to ensure mutual exclusion for that other thread. Otherwise (lock is free, (3), the transaction enters the actual body of the atomic section.

The transaction is executed speculatively by the TM hardware while checking for aborts. If it completes all of its work, the thread executes the commit operation/instruction, causing the TM hardware to atomically write to non-speculative state all of the data that was speculatively written. If an abort does occur, the speculative state is automatically discarded (4) and the thread switches control to the abort handler, which can retry the transaction $(\boldsymbol{5})$ or use the fallback path for it (6).

\section{PRE-ABORT HANDLERS}

In Figure 1, note that the speculative state is automatically discarded when an abort condition is met. For aborts caused by data conflicts, this is a reasonable approach. Data conflicts can lead to partial work being exposed and atomicity being violated. However, in many cases, the transaction is aborted because it cannot continue, discarding the work done so far even though that work is still valid. For example, a transaction that overflows the speculative buffer cannot continue because, if a data conflict does occur later on, the transaction's writes can no longer be rolled back.

This does not mean the work must necessarily be discarded. For example, when a transaction overflows the speculative buffer, the other threads could be paused by a hardware mechanism [4] to allow the overflowing transaction to complete correctly. The same effect can be achieved by atomically converting the transaction into 
an irrevocable transaction just before it overflows the speculative buffer. However, it is difficult to do so by anticipating the overflow in the transaction's own code [32]. Reactive approaches (conversion when buffer overflow is just about to occur) are precluded by the TM hardware automatically aborting the transaction without giving the software any opportunity to remedy the situation and possibly avoid the abort.

As a solution, we propose to allow the hardware TM to invoke a function, a pre-abort handler, when the transaction encounters an abort-causing condition (in other words, before 4 in Figure 1). This will allow the programmer to decide whether to take mitigating action to avoid the abort-causing situation, or keep the default behavior and discard the speculative work.

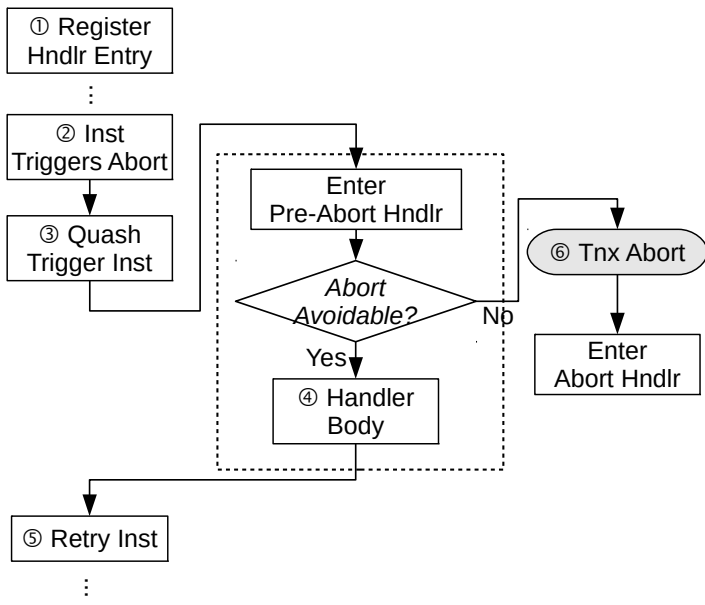

Figure 2: Flowchart With Pre-abort Handler

Figure 2 depicts a high level view of the steps taken. Before we start the transaction, the entry point of the handler function is registered to the HTM system (1). Later, when the transaction encounters a situation that can trigger an abort (2), say a capacity overflow, we treat it as if the instruction had an exception; the instruction is quashed and the PC value of the trigger operation is saved (3). Note that unlike typical exceptions or interrupts on processors that support HTM, this exception does not itself trigger an abort.

The thread is then diverted to the handler entry point. The handler is provided arguments such as the type of abort-causing situation, which can be used to decide which mitigating action to take (4)). After the situation has been resolved, the handler returns. The PC of the trigger operation is restored, allowing the thread to re-attempt the operation (5)). Hopefully, the abort-causing condition has been fully resolved, and the transaction is no longer threatened.

In some cases, the situation is unresolvable and the transaction has no other option but to abort. In this case, the handler code calls abort explicitly (6), causing the thread to follow standard abort handling procedure (i.e. abort and rollback the transaction, then enter the abort handler).

The fact that the pre-abort handler was triggered does not mean that the transaction is immune from abort. Other abort-causing conditions, such as regular data conflicts, are still tracked. If there is an additional abort causing condition during execution of the pre-abort handler (nested aborts), the transaction is aborted and speculative state discarded, with the program control passed directly to the (regular) abort handler. Pre-abort handlers must be carefully written, since they execute non-speculatively, but may be interrupted (and never continued) at any time.

\subsection{Supported Abort Types}

Table 1 shows what abort types we handle, and what additional arguments are passed (if any).

Table 1: Abort Types with Pre-abort Handlers

\begin{tabular}{ccc}
\hline Cause & Handled? & Additional Arg \\
\hline Syscall & Yes & Number \\
Capacity & Yes & None \\
Manual Abort & Yes & Abort Argument \\
Conflict on Clean & Yes & Conflict Address \\
Conflict on Dirty & No & N/A \\
\hline
\end{tabular}

When a system call is attempted we call the pre-abort handler instead of aborting. The handler is passed syscall as the cause argument, and an additional argument, the system call number.

When a transaction hits its capacity limit, the handler is called with the cause argument as capacity.

Many HTMs allow transactions to be aborted explicitly, e.g. by executing an XABORT or TABORT instruction [6, 17], along with a user-defined abort-cause argument. We also call the pre-abort handler in this case. The user-provided argument is passed as the additional pre-abort argument. In this case, the handler is set to return to the instruction after the abort instruction (as opposed to returning to the instruction itself), since it makes no sense to retry the instruction and return right back to the handler.

The pre-abort handler is also invoked for conflicts on lines that were only read by a transaction handler (transactional and clean). In this case, the virtual address of the cache block that experienced the conflict is also supplied to the pre-abort handler. This is somewhat similar to interrupts from Alert-on-Update[27]. However those interrupts focus on monitoring the state of software TM metadata, and are not for general HTM.

However, a data conflict on a transactional but dirty line implies that the transaction has already done work that, if committed, could violate transactional semantics (unless there was false sharing, which is outside the scope of this paper, or unless the conflict resolution policy is changed). Therefore, in this case we allow the HTM to automatically abort without pre-abort handler invocation, but we envision this behavior to be software-configurable to permit further uses of pre-abort handling, e.g. to avoid false-sharing-induced aborts.

\subsection{Hardware Support}

To support pre-abort handlers, the first hardware change that is needed is a mechanism to provide the processor core with the handler's address. One option is to reuse existing interrupt/exception vector tables and user-space signal handler mechanisms. Another 
way, which we use in our evaluation, is to add a new register [22], which we call the Pre-abort Handler Entry register. To put the address of the handler into this register, we can use an existing instruction for accessing control registers or, if no such instruction exists, introduce such an instruction.

The hardware already must track whether a thread is inside a transaction or not, e.g., to control the behavior of reads and writes. We add a third possible state, HANDLER. We change the hardware such that, when an abort-causing condition is detected by a thread in the TRANSACTION state, it doesn't automatically discard speculative state and transition to the NON-TRANSACTIONAL state. Instead, it saves the PC and transitions to the HANDLER state.

As the system enters the HANDLER state, the stack pointer is also advanced by the cache line size to prevent the handler's nonspeculative accesses from interfering with the transaction's speculative data. Without such precautions, the handler and the suspended transaction may end up sharing the same cache line (the top of the stack). This will result in the line being in some sort of anomalous state.

When entering the pre-abort handler, the PC is set to the entry point of the pre-abort handler (effectively jumping to that instruction). The arguments of the handler function need to be set as well, which we do so by following function calling convention and setting R1 to the abort cause and R2 to the abort-specific additional argument.

While in the HANDLER state, memory is accessed speculatively or non-speculatively depending on the status of the cache line. Reads or writes to cache lines with the transactional bit set are done speculatively, whereas non-transactional lines and lines not being tracked yet (e.g., cache misses) are done non-speculatively [8, 9]. In other words, the transactional bit is left as-is for each access ${ }^{2}$.

The eventual fate of the handler can be one of three things. First, if the handler returns without the transaction getting committed or aborted, the transaction is resumed. The saved PC is restored and the stack pointer is moved back one cache line. If the abort was due to a clean data conflict, the line is added back to the read set. Lastly, the transaction state is changed from HANDLER to TRANSACTION.

Second, the handler may call the transaction commit instruction. In this case, the thread commits all speculative state as usual and moves from the HANDLER state to the NON-TRANSACTIONAL state. However, the thread has not yet left the handler function; returning triggers the same stack pointer and PC adjustment as in the "resume" case.

Lastly, the thread can encounter another abort-causing condition while still inside the pre-abort handler (nested abort). For example, while handling a system-call event a data conflict occurs. In this case, we make no attempt to handle this nested abort situation and take the normal abort path (i.e. discard speculative content and call the regular abort handler).

In summary, while in the HANDLER state, the hardware behaves as if it's executing a transaction, except:

- The stack pointer is advanced and returned when entering and exiting the handler.

- Detecting additional abort causing conditions will abort the transaction.

\footnotetext{
${ }^{2}$ This means that the handler can silently modify speculative state.
}

- Accesses to cache lines not yet tracked as part of a transaction's read set or write set are done non-speculatively.

The HANDLER state is similar to transaction suspension provided by IBM's Power architecture [6]. However, in our scheme the suspension is done as part of the pre-abort handler, instead of being done explicitly.

It is important to note that the changes proposed above are isolated within the processor core itself, including both the TM hardware and private cache, and no changes are needed for the coherence protocol. In addition, if the entry point is not registered, there will be no handler call. The program behavior in this case is identical to the baseline: speculative state is discarded and control passed to the (regular) abort handler, allowing for backward compatibility.

In the following sections, we describe various use cases of the pre-abort handler.

\section{PRE-ABORT HANDLER USE CASES}

\subsection{Handling System Call Aborts}

Including system calls inside transactions is problematic because they are hard or impossible to undo [28]. As a result, conventional HTM systems $[17,18]$ simply abort when encountering system calls, forcing the transaction to discard the work done so far and take the fallback path. The thread then re-executes the code before the system call, although now inside a critical section.

However, system calls are highly useful in debugging and for other purposes [33]. Indeed this is one reason why the C++ Transactional Memory proposal included the

optimize-for-synchronized keyword to handle transactions that include system calls. Without them, a TM-aware compiler will fail to generate transactions and use critical sections instead [30].

By using pre-abort handlers, instead of automatically aborting, we can atomically convert the transaction into a critical section. Specifically, the handler is invoked when a transaction attempts a system call. This handler then attempts to acquire the fallback lock. Because all transactions hold the lock status in their read sets (1 in Figure 1), the acquisition will force the other threads to abort any active transaction and prevent them from starting new ones. Once the lock is acquired, the thread then commits its work in isolation and returns to the system call instruction. As a result, the transaction's already-performed work can be considered to logically happen inside the critical section. At the end of the (now) critical section, the overall state of the thread is equivalent to what would have happened if the fallback path was used instead of starting the transaction.

This conversion of transactions into critical sections is similar to Irrevocable Transactions [29] that were used in the context of a lockbased software transactional memory (STM). However, instead of relying on the STM implementation's centralized contention managers and software locking protocols, pre-abort handlers provide similar benefits for best-effort HTMs.

Figure 3 shows the code, somewhat simplified for clarity, of how we might implement this idea using pre-abort handlers. When a transaction encounters a system call instruction, under the baseline 
Transactional Pre-abort Handlers

in Hardware Transactional Memory
PACT '18, November 1-4, 2018, Limassol, Cyprus

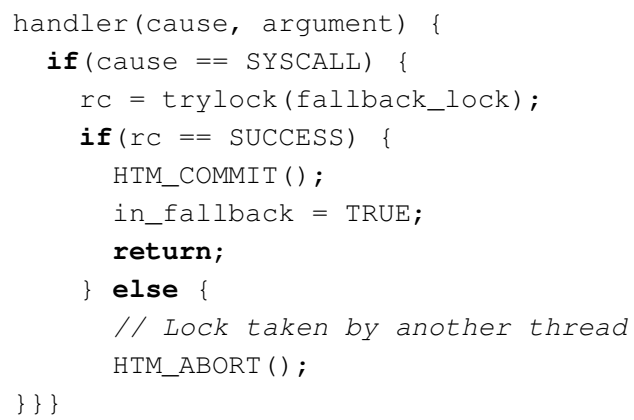

Figure 3: Pre-abort Handler For System Calls

system, the transaction is aborted and speculative state automatically rolled back. With pre-abort handlers, the instruction is instead quashed, just as is done for ordinary exceptions. The thread's control is then passed to the pre-abort handler.

The handler code first checks the reason for the exception (line 2) and then requests the fallback lock (line 3 ). When the lock is acquired and transaction successfully committed (line 4 - line 6), the transaction can no longer conflict with other transactions. The fallback lock is acquired and held by our thread, so active transactions are aborted and new ones prevented from starting. Since speculative state is also committed, the thread is now outside of a transaction, and the thread's state is as if it had been executing all along as a critical section.

When the thread returns from the handler, it continues executing non-speculatively, starting with the system call operation that originally triggered the conversion. The thread continues execution until the end of the "critical section," at which the fallback lock is released, just like it would be if the critical section had been executed using the fallback path in the first place.

Note that it is possible for the lock acquisition to fail, because the lock was already acquired by another thread (line 8). In this case, the pre-abort handler simply aborts, which leads to the speculative state being aborted and the regular abort handler being called.

It is even possible (but extremely rare) for a thread to successfully acquire the lock, but get aborted before the transaction was able to commit. For example, a conflicting access in another thread may occur just before the handler reaches the commit point. Since transactions inside the pre-abort handler are still vulnerable to data conflicts, the transaction will simply be aborted, and control diverted to the regular abort handler. The thread still holds the lock (non-speculatively), so the regular abort handler simply needs to start the critical section body.

In all of these unsuccessful conversion cases, although the thread's speculative work is not salvaged, the overall resulting behavior is correct; in fact, it is the same behavior that occurs without transaction conversion.

If needed, we can also check the cause-specific argument, which according to Table 1 is the system call number, and take different action depending on which system call was the trigger. However, we choose not to do so in this implementation.
One advantage of our solution is that transaction conversion does not need to be done ahead of time. Other solutions [6, 29] require code modification that inserts transaction conversion just before the system call is attempted. However, as we shall see next, there are other types of non-conflict aborts that are more difficult to predict.

Another advantage of our solution is that when a transaction encounters a system call, the transaction does not need to discard work and retry. This is because in some cases system calls are issued due to a rare condition being triggered, perhaps an error condition. However, the condition may no longer be valid when the transaction was aborted and restarted as a critical section. By allowing the transaction to seamlessly convert into a critical section right then, the program can be in better shape to act on the situation.

\subsection{Handling Capacity Aborts}

When a core executing a transaction runs out of buffer space, the transaction is unable to proceed further without compromising its ability to abort and roll back. In this case, the cache-overflowing transaction is forced to abort before proceeding further. These are called capacity aborts. Capacity problems have been observed to be a major cause of aborts in commercial HTM systems [31], and an obstacle to broader use of HTM [5].

In addition, capacity aborts can be especially detrimental to performance. Existing best-effort HTM systems handle capacity aborts in the same way as system call aborts: the fallback path is taken. The fact that a transaction has accumulated enough read or write addresses to overflow buffer capacity means that it has probably done a lot of work. All that work is discarded and re-done on the fallback path, simply because of a limitation in the hardware. Even though the transaction has not conflicted with another thread and the already-done work is correct up to that point. If we instead have a mechanism to catch the capacity overflow before it occurs, we can take abort-avoidance action (e.g. convert the transaction into a critical section), we can avoid discarding the work.

However, unlike with system calls, which have a clear point in the code where it is clear that an abort-causing situation is going to take place, capacity issues are more difficult to predict. For system calls, this means that mitigating action can be inserted just before the system call through manual inspection [29], or even through some type of compiler analysis.

For capacity aborts, any memory access may be a possible abort site, depending on the size and other characteristics of the transactional state buffer (e.g. the private L1 cache), and the actual mix of addresses accessed by the transaction, etc. [8, 32]. This type of interaction is implementation dependent and hard to predict ahead of time - in many ways this problem is similar to the cache performance analysis problem, which is usually measured at runtime [24] rather than predicted statically.

This makes our handler-driven mechanism much more important. Instead of relying on a prediction mechanism that may or may not be correct, the pre-abort handler is only triggered when the buffer capacity overflow is actually imminent. Other than including a check for capacity abort, the pre-abort handler for transaction conversion just before a capacity abort is very similar to the one for system calls (Figure 3). 


\subsection{Inserting Non-transactional Work}

Another use case of pre-abort handlers is to include non-transactional 2 work within a transaction. One challenge with debugging hardware transactions is due to isolation provided by the hardware [33]. Even if there is a bug within the transaction, the state is discarded on abort, lost to the developer.

If there was a mechanism to log thread state non-transactionally, this state will still be available after the abort, making it easier to write correct transactions. This can be done by either adding special memory instructions [9] that execute non-speculatively, or instructions that suspend and resume speculative execution [6]. Unfortunately, these proposals require special hardware changes.

In comparison, the same pre-abort handler mechanism can also be used to provide such functionality. To do so, the developer manually triggers the pre-abort handler, by calling the XABORT or TABORT instructions. The argument passed to this abort instruction is passed as an additional argument to the handler (see Table 1). This can be used to pass information about the current transaction to the handler. Section 5.2 contains an example handler, which nontransactionally logs actions done by the active transaction, which can be inspected later on.

\subsection{Pausing Transactions on Fallback-lock Activity}

When a thread is using the fallback lock, the correct behavior is to disallow other transactions from executing, either by aborting or pausing them. This is because the critical section executes nonspeculatively and does not rely on buffering to provide isolation. If other transactions are allowed to continue, this will result in undetected conflicts, which would violate transactional guarantees, as described in Section 2. However, other in-flight transactions have not (yet) conflicted with the critical section, so aborting them can be inefficient.

Instead, it is actually sufficient to pause the other transactions, rather than abort them immediately [14]. When the other transactions are notified of the fallback lock being acquired, they are paused, prevented from doing any additional work that can update the read or write sets. Only when the critical section is completed and the lock released are the transactions allowed to complete.

By using pre-abort handlers, we can implement transaction pausing. We use the fact that the pre-abort handler can be called when transactional but clean data is written to. Since fallback locks are only read (at the beginning of the transaction) but not written to, when there is lock activity, this will lead to the pre-abort handler being triggered. Transactions can then wait inside the handler, waiting for the lock to become free again. Once the lock is freed, the paused transactions are allowed to return from the handler, and resume operation.

Figure 4 depicts a handler that handles conflicts on the fallback lock. The transaction, as shown in Figure 1, starts by testing the fallback lock status and adding the address to the transaction read set. Before the transaction completes, the HTM hardware is notified of a write to a cache line that was part of its read set. The current operation is quashed and the pre-abort handler is triggered.

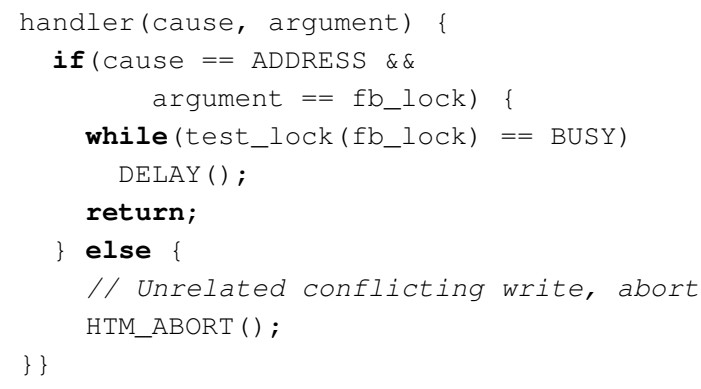

Figure 4: Pre-abort Handler for Fallback-lock Conflicts

Since the handler is passed the address of the data conflict, we use this to see if it matches the address of our fallback lock (line 2line 3). If the address does not match (line 8), this means the preabort handler was triggered by a write to another address. This is a genuine data conflict, and the pre-abort handler aborts the transaction right away (line 10).

If the address does match, this means there was a change to the lock status (line 4). The handler loops until the busy lock is now free, effectively pausing the transaction (line 4 - line 5).

Once the lock becomes free, the loop ends and the handler returns (line 6). The PC is now back at the original operation and resumes normal execution. The transaction resumes, as if nothing has happened.

Pausing transactions, rather than aborting them, still results in correct execution. As noted earlier, a transaction paused within the pre-abort handler is still vulnerable to data conflicts. A paused transaction can have a data conflict with the critical section in one of two ways: with data that transactions had accessed before the lock acquisition, or after the lock release.

The first type of data conflict occurs when data the transactions had accessed is later accessed by the critical section. In this case, this is a standard type of data conflict; if the conflict was on dirty data, the transaction is automatically aborted, if it was on clean data, this is a nested abort and the transaction will (again) be aborted.

The second type of data "conflict" occurs when the data accessed by the critical section is later accessed by the transaction. In this case there is no atomicity violation because such execution is equivalent to serialized execution of these transactions in completion-order: first the critical section completes and releases the lock, afterwards, paused transactions eventually commit, after the critical section.

An important property we are taking advantage of is the fact that lock states are reversible; a lock that is busy will later be released and become free. Transactions are paused only while the fallback lock is busy, but when the fallback lock is freed, they are allowed to resume.

When the fallback lock shares a cache line with other data or locks, this can result in many more data conflicts being detected. Although this can hurt performance, this does not mean the program is incorrect. Transactions will be unnecessarily aborted or have their pre-abort handlers triggered unnecessarily. The test_lock operation will return that the lock is free, and the transaction will be allowed to continue, albeit delayed somewhat. 
Transactional Pre-abort Handlers

in Hardware Transactional Memory

One might be tempted to take this idea further and allow transactions to continue, blocking only when they try to commit. This is called lazy lock subscription, as opposed to early subscription which is what we use. However, lazy subscription can lead to problems [11]. Specifically, note that the critical section runs non-speculatively, updating values immediately. If the other transactions are not paused, they will be observing inconsistent state, breaking atomicity of the critical section. Since this can lead to further incorrect behavior, we avoid taking this route and simply pause the other transactions while the fallback lock is held.

\subsection{Pausing Transactions on Critical Section Conversion}

Interestingly, pausing transactions can be combined with converting transactions into critical sections. In Figure 3, when a transaction is converted into a critical section, the other active transactions are aborted immediately. This is done to keep the relationship between fallback lock activity and transactions identical: when the lock is acquired all active transactions are aborted.

Sometimes, multiple transactions can request being converted into a critical section at the same time. However, under the previous version of the pre-abort handler, only the first transaction succeeds; the others are aborted. Instead of doing so, we can try to pause them instead. When multiple transactions need to be converted into a critical section, we allow each of them to do so (one after the other), instead of needing multiple attempts before finally running as one.

We can do so through a simple change to the handler in Figure 3. Instead of trying and giving up after the first attempt, lock acquisition is attempted repeatedly. The successful thread will be converted into a critical section, while the other threads will be paused, waiting for the lock to become free again (or be aborted while waiting)

\subsection{Other Possibilities}

In addition to the cases discussed so far, one can think of additional possibilities for pre-abort handlers. For example, one might use such pre-abort handling to avoid transactional aborts due to "housekeeping" activity, such as maintaining thread-local memory pools. When the memory pool is exhausted, accessing the global pool can lead to data conflicts. Instead, the transaction can be suspended while the thread-local free memory pool is replenished from the global pool. This is safe because even if the transaction is aborted, this expansion of the local free memory pool is safe (and usually beneficial) to keep rather than undo.

As another example, we can implement transaction early release with pre-abort handlers as well [16, 23]. Early release allows transactions to drop addresses from its read set, allowing the transaction to ignore conflicts on such addresses. We can do so by maintaining a per-transaction set of "dropped addresses." Later, when there is a read-write conflict interrupt and the addresses are confirmed to be part of the dropped addresses set, the conflict can be ignored, allowing the transaction to continue.
PACT '18, November 1-4, 2018, Limassol, Cyprus

\section{EVALUATION SETUP}

We evaluate pre-abort handlers using SESC, a cycle-accurate architecture simulator [26], with an HTM model based on SuperTrans [25] modified to more closely match Intel's TSX. The processor cores are 4-way out-of-order, with private L1 and L2 caches. The L1 cache is $32 \mathrm{~KB}, 4$-way set-associative, with hit latency of 4 cycles. The L2 is $64 \mathrm{~KB}$ per core with a hit latency of 18 cycles. The shared L3 cache is $1 \mathrm{MB}$, with a hit latency of 34 cycles. The memory takes a constant 200 cycle latency.

Each L1 cache block is augmented with a bit indicating its transactional status. If a line with the bit set is to be evicted from the cache set, this is a capacity overflow. If it receives a coherence request, this indicates a data conflict.

When a transaction encounters an abort-causing condition, the hardware checks if the pre-abort handler entry point is set. If it is, the program control is switched to the pre-abort handler. If not, the transaction is aborted immediately and program control switched to the regular abort handler. If the pre-abort handler decides to give up, the transaction is also aborted and program control switched to the regular abort handler. The abort penalty we used is a pipeline flush.

Three different configurations are studied. The Baseline configuration does not have the pre-abort handler specified and aborts on system calls, capacity limits, and fallback activity. Hndl uses a pre-abort handler to avoid system call aborts and capacity aborts by converting the transaction into a critical section (see Section 4.1 and Section 4.2). Hndl+ additionally avoids aborting on fallback lock activity by pausing transactions (Section 4.4 and Section 4.5). In all three configurations, the regular abort handler uses random linear backoff, and takes the fallback path after 12 conflict aborts or 1 system call abort or capacity abort.

In Section 5.1, we first use microbenchmarks to demonstrate the utility of using pre-abort handlers. The first microbenchmark studies the impact of system call aborts and the benefits of handling them through pre-abort handlers. The second microbenchmark studies the impact and benefit of handling capacity aborts.

We then look at an application, vacation, from STAMP [23] and add non-transactional logging using pre-abort handlers, and quantify the overhead required.

Lastly, we use the STAMP benchmarks in section 5.3 to further quantify benefits on capacity aborts and fallback lock activity aborts; STAMP does not include system calls or non-transactional work within transactions. We exclude kmeans and ssca2, because their read/write set sizes are small and constant regardless of input, limiting the number of capacity aborts we can catch. In addition, they do not have many aborts in the first place and would not benefit from fallback activity mitigation techniques either. For measuring performance, we used the "Time" output from the program itself, which shows the wall clock time of the application's parallel region.

\subsection{Microbenchmark Results}

We first use microbenchmarks to show what benefits can be realized using our abort mitigation techniques. In Figure 5 we see the results from the first microbenchmark, which has transactions that may issue system calls. An AVL tree with objects, each 64-bytes large (1 
cache line) and with a unique search key, is generated. A list of random search queries is generated as well, with a $50 \%$ probability of missing, and distributed to each thread. Each thread issues (readonly) search queries against the tree, and when the key is not found, an error message is logged to stdout. We compare the throughput of each of the three configurations.

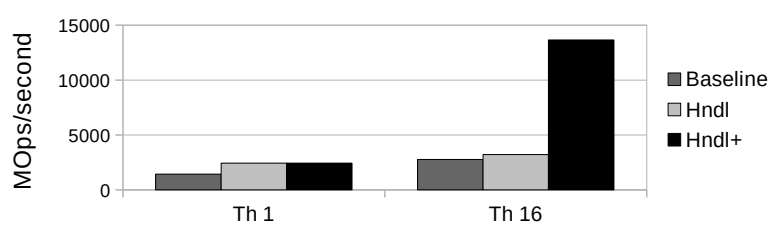

Figure 5: System Call Microbenchmark Results

From the results, we can see that there is a $70 \%$ speedup when using transaction conversion compared to the baseline when running single-threaded. This is because the microbenchmark issues a system call at the end of a transaction when it fails to find the query key. This means that a failed query will result in the thread executing a transaction almost to the end, abort due to the system call, and re-attempt the same code all over again.

At 16 threads, even though we have the threads all attempting transactions, under Baseline many of them eventually issue system calls and trigger further aborts on the fallback path. Unfortunately for $\mathrm{Hndl}$, converting a transaction still results in the other transactions getting aborted, even if none of them encountered any conflicts. By pausing these other transactions instead, Hndl+ allows the microbenchmark to achieve a total of $9.5 \mathrm{x}$ speedup over the single-threaded result.

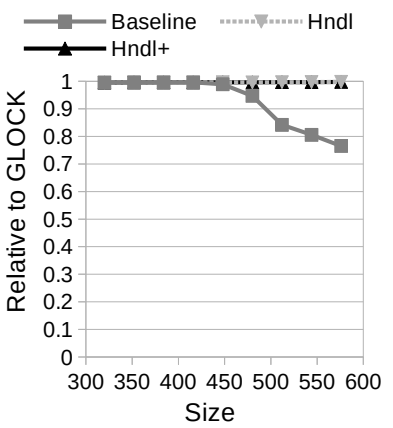

(a) 1 Threads

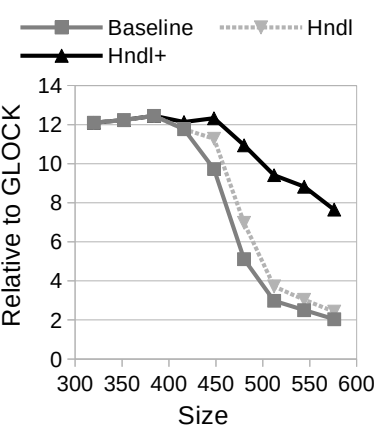

(b) 16 Threads
Figure 6: Capacity-scaled Microbenchmark Results

In Figure 6 we see the results from the second microbenchmark, which demonstrates capacity aborts. The microbenchmark has a randomly generated array along with a sequence of search queries generated beforehand. Similar to the previous microbenchmark, each entry is 64-bytes, and the search query has a $50 \%$ chance of missing. Each thread performs linear searches through the shared array, with one search per transaction. A larger search key will force the transaction to read more of the array and eventually run up against the capacity limit. We compare the throughput of the three configurations against GLOCK, which uses a single global lock instead of a transaction and therefore does not have any capacity issues.

When running with 1 thread, as we scale the input size, the performance of Baseline drops significantly, whereas the other two configurations are able to keep up with the global lock version. As we go from an input size of 448 to 576, Baseline suffers from a performance dropoff of no performance loss to almost $24 \%$ performance loss due to increased capacity aborts. Using pre-abort handlers, we are able to salvage the work done by the overflowing transaction, and recover most of that performance loss, a $30 \%$ increase over Baseline.

When running with 16 threads, the impact of capacity overflow aborts becomes more prominent. In the same range of 448 to 576, Baseline goes from providing a 12x speedup over GLOCK down to $2 \mathrm{x}$ speedup, a 6 -fold performance drop. If we allow transactions that hit the capacity limit to be converted into a critical section, the impact is reduced, resulting in a $10-30 \%$ performance improvement over Baseline. If we are able to pause them instead, as with $H n d l+$, we are able to greatly reduce the impact of the performance drop. The reason is because when one transaction hits the capacity limit, we are able to preserve the others by pausing instead.

In addition to improving performance for transaction sizes that are past the capacity limit, pre-abort handlers are able to provide a more graceful degradation just past that threshold.

\subsection{Non-transactional Work Results}

We investigate the benefits of including non-transactional work within transactions by modifying the vacation application from STAMP. Vacation simulates a travel reservation system, and randomly issues requests to an in-memory database: make reservation, delete customer, add and remove from item tables. We add a log entry to every customer deletion operation, as shown in Figure 7.

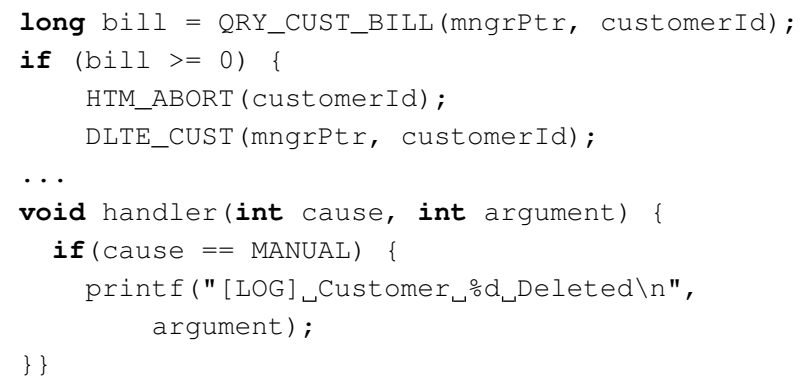

Figure 7: Adding non-transactional logging to vacation

The manual abort is provided a customer Id argument, which is then passed on to the pre-abort handler. The handler then logs this information, non-transactionally to standard output. Running with the simulator input, of the 4096 requests, $5 \%$ of the them resulted in a customer delete request. Logging these requests resulted in $2.0 \%$ runtime overhead, which is quite limited. 


\subsection{STAMP Results}

Figure 8 show the overall performance improvement provided by our mechanism when running STAMP, running with recommended simulator input. Since STAMP does not issue system calls within transactions, our experimental setup will only help on capacity aborts and fallback lock activity. We compare the execution times of the region of interest between Baseline and Hndl+.

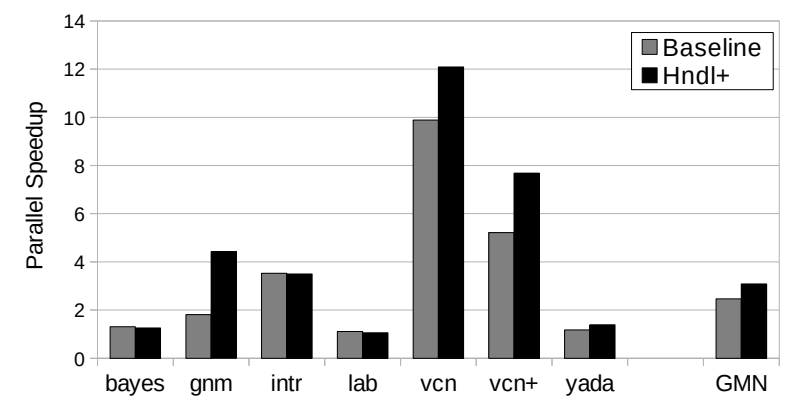

Figure 8: Overall Results

Using pre-abort handlers to convert transactions that overflow capacity, and pause them upon fallback lock activity, Hndl+ shows an average speedup of $25 \%$ over Baseline. Genome sees the most benefit (2.4x speedup). This is because a significant portion of its abort cycles are attributable to capacity-related issues, either directly because a transaction hits its capacity limit and aborts, or indirectly because capacity-aborting transactions later take the fallback path and abort other non-conflicting transactions. Vacation and yada also shows good gains (22\%, $47 \%$, and $18 \%)$.

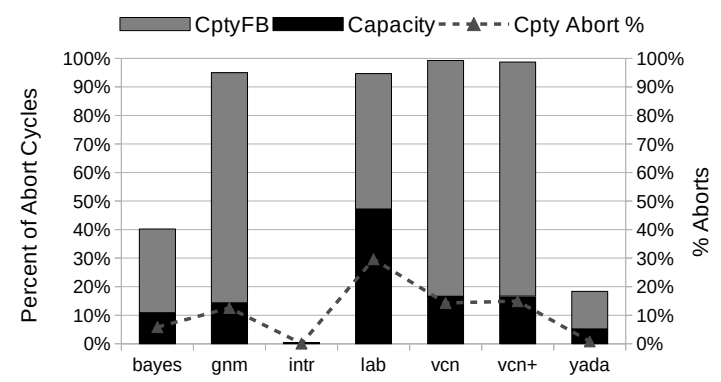

Figure 9: Contribution of Capacity Aborts

Figure 9 shows the percentage of all aborted cycles that can be attributed to capacity overflow when running the STAMP benchmarks with the baseline system. Capacity shows cycles directly attributable to capacity aborts, i.e. cycles spent reaching the capacity abort, as a percentage of all aborted-execution cycles (due to capacity, conflicts, etc.). Cpty FB shows the cycles indirectly attributable to capacity aborts: work lost in other transactions as they are aborted when the capacity-aborted transaction enters the fallback path. Cpty Abort \%, shows the percentage of all transaction aborts that are directly attributable to capacity overflows (in other words, transaction attempts in this category contribute to Capacity cycles).
As we can see, in applications that do experience capacity overflow, the impact to lost work (cycles) is consistently larger than the fraction of capacity overflow aborts. This means capacity aborts are typically more costly than other aborts. Capacity aborts come from a transaction having done a lot of work, even too much work, which is then discarded. In contrast, conflict aborts may happen very early in a transaction. The sole exception is labyrinth; capacity overflows occur so early in the transaction that threads are serialized and the benefit is minimal.

Interestingly, the results also show that that a significant fraction of aborted cycles are indirectly attributable to capacity overflows. These aborts are caused by the fallback path being taken by overflowing transactions. As noted earlier, in the baseline system, transactions are required to abort when the fallback path is active, to ensure atomicity.

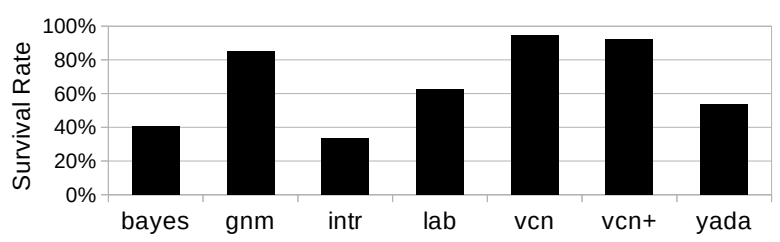

(a) Survival Rate

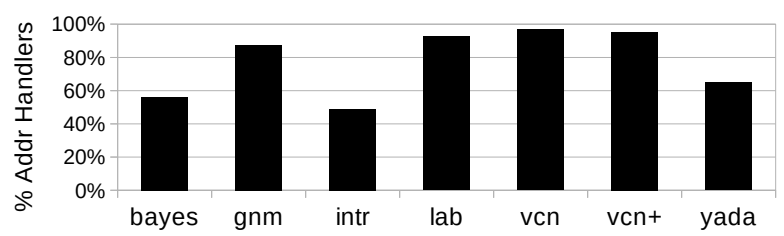

(b) Pre-abort Handlers Caused by Fallback Lock Activity

\section{Figure 10: Other Statistics}

Figure 10a shows the survival rate of paused transactions, i.e. the fraction of paused transactions that are eventually resumed (as opposed to being aborted while paused). A high survival rate indicates that the active thread (a critical section or a converted transaction) is less likely to conflict with paused transactions. These benchmarks can be expected to show increased benefit to those that have a lower survival rate. Indeed, genome and vacation have very high survival rates, and therefore see more benefit from pausing. Other benchmarks have lower survival ratios and their benefit from pausing is lower.

Figure 10b shows what percentage of pre-abort handler activity was triggered by the fallback lock, compared to the total number of clean conflict handler calls (writes to transactional but clean data). Benchmark applications that have a high ratio means most pre-abort handlers were actually due to fallback lock activity. This is the case for many of our STAMP applications, although we can see that in bayes, intruder, and yada we see that many address handler invocations are due to actual data conflicts instead.

Because we call the pre-abort handler instead of automatically discarding a transaction's state and aborting it, one might be concerned about the overhead caused by the handler. To measure this overhead, we compared the runtime of the baseline implementation 


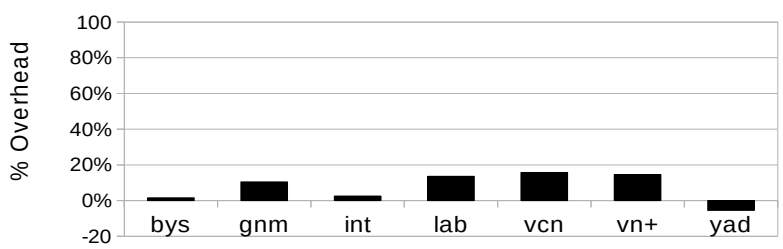

Figure 11: Overhead of Pre-abort Handlers

(automatically roll back the transaction) with an empty pre-abort handler (in other words, the pre-abort handler doesn't do any mitigating action and always aborts).

The results are shown in Figure 11, which shows that the overhead of invoking a pre-abort handler is relatively small and is easily outweighed by the performance benefits enabled by these handlers.

\section{RELATED WORK}

Alert-on-Update [27] describes a mechanism that triggers an interrupt when a cache line is evicted from the private cache. It relies on new instructions that adds a cache line to an alert set, which can cause an interrupt handler to be called when written to. Unlike our pre-abort handler, Alert-On-Update's handlers are limited to monitoring its software TM metadata.

Irrevocable Transactions $[21,29]$ is an STM system that converts a transaction into a special irrevocable mode. When a transaction is converted to this mode, it always has highest priority in data conflict resolution. At a high level this is similar to our transaction conversion. However, because it targets lock-based STM systems, the changes required are simpler. In addition, it does not need to support transaction capacity issues, which is difficult to predict without using pre-abort handlers.

Even though they are enclosed within a transaction, many memory operations are not required to be marked as speculative and be tracked by the HTM system. Some examples are read-only data or private stack data. Instead of treating all memory operations as speculative, some architectures such as ASF allow the software to selectively choose which operations are speculative, and which are non-speculative $[8,9]$.

Transaction Suspension [6] is a mechanism in Power's implementation that allows speculative execution to be temporarily suspended. Although this can also be used to take mitigating action against certain type of aborts, this requires the transaction to predict the abort situation and preemptively take action.

Between All and Nothing [12] proposes transactional resurrection, which allows aborted hardware transactions to resume. However, their default mechanism only steps in after the working set is cleaned up. The system then replays the software log and "resurrects" the aborted transaction. There is also mention of a "messy" version, which does not discard the working set (like with preabort handlers). However, it is only intended only intended for short delays, such as TLB misses, and is not discussed further.

System calls are challenging to support within transactions due to the side effects involved [1]. xCalls [28] allow transactions to use system calls, but require extensive software changes to work.
FasTM [20] tracks transaction metadata in hardware, but falls back to the software log when a transaction exhausts its capacity. However, this requires the hardware understand how to manage the log and add an additional coherence state, which makes it less attractive for commercial HTM systems.

OneTM [4] introduces an overflowed state, which stalls other transactions while the first transaction attempts to complete. Conceptually, this approach is similar to paused transactions with transaction conversion, but requires transaction logging in software. TCC [14] is another approach related to transaction pausing. It allows other transactions to continue when a transaction encounters a capacity limit, blocking only their commit. However, commit blocking has problems similar to lazy subscription. In addition, TCC also requires a commit arbiter, which is not available in conventional HTM.

Proactive Transaction Scheduling [3] and Preventing versus Curing [13] model the probability of conflict and use this to guide a transaction scheduler. Similarly, Bloom Filter Guided Transaction Scheduling [2] use predicted read and write sets to avoid likely conflict aborts. These proposals focus mainly on predicting and avoiding data conflicts, and is largely orthogonal to our work.

Hybrid transactional memory [7, 10, 19] augments transactions running on conventional HTM with software TM. A transaction can decide before starting whether to run as software TM or hardware TM, and care needs to be taken to make sure data conflicts between transactions in either mode are properly tracked. It will be interesting to see whether pre-abort handlers can help hybrid TMs in this regard, but we leave this as future work.

\section{CONCLUSIONS}

Commercial HTM offerings are becoming common. However, there is room for improvement, especially related to the cost of aborts. When transactions are aborted, speculative state is automatically discarded and the thread rolled back when an abort-causing situation is encountered. This is the case even when the state is still perfectly valid.

Pre-abort handlers provide the programmer an opportunity to intervene when an abort-causing situation is encountered. When inside the non-transactional handler function, mitigating action can be taken to avoid the abort, or non-transactional work can be included, which has previously required custom hardware support. Pre-abort handlers support such additional functionality, through a simple addition to best-effort HTM.

\section{ACKNOWLEDGEMENTS}

This work has been supported, in part, by NSF grants CCF-03200717 and CCF-1563991. The views and findings in this paper are those of the authors, and do not necessarily reflect the views of NSF.

\section{REFERENCES}

[1] L. Baugh and C. Zilles. 2008. An Analysis of I/O And Syscalls In Critical Sections And Their Implications For Transactional Memory. In Performance Analysis of Systems and software, 2008. ISPASS 2008. IEEE International Symposium on. 54-62. https://doi.org/10.1109/ISPASS.2008.4510738

[2] G. Blake, R.G. Dreslinski, and T. Mudge. 2011. Bloom Filter Guided Transaction Scheduling. In Intl. Symp. on High Performance Computer Architecture (HPCA '11). 75-86. https://doi.org/10.1109/HPCA.2011.5749718 
[3] Geoffrey Blake, Ronald G. Dreslinski, and Trevor Mudge. 2009. Proactive Transaction Scheduling for Contention Management. In IEEE/ACM Intl. Symp. on Microarchitecture (MICRO 42). 156-167. https://doi.org/10.1145/1669112.1669133

[4] Colin Blundell, Joe Devietti, E. Christopher Lewis, and Milo M. K. Martin. 2007. Making the Fast Case Common and the Uncommon Case Simple in Unbounded Transactional Memory. In Proceedings of the 34th Annual International Symposium on Computer Architecture (ISCA '07). ACM, New York, NY, USA, 24-34. https: //doi.org/10.1145/1250662.1250667

[5] Jayaram Bobba, Neelam Goyal, Mark D. Hill, Michael M. Swift, and David A Wood. 2008. TokenTM: Efficient Execution of Large Transactions with Hardware Transactional Memory. In Proceedings of the 35th Annual International Symposium on Computer Architecture (ISCA '08). IEEE Computer Society, Washington, DC, USA, 127-138. https://doi.org/10.1109/ISCA.2008.24

[6] Harold W. Cain, Maged M. Michael, Brad Frey, Cathy May, Derek Williams, and Hung Le. 2013. Robust Architectural Support for Transactional Memory in the Power Architecture. In Proceedings of the 40th Annual International Symposium on Computer Architecture (ISCA '13). ACM, New York, NY, USA, 225-236. https: //doi.org/10.1145/2485922.2485942

[7] I. Calciu, J. Gottschlich, T. Shpeisman, M. Herlihy, and G. Pokam. 2014. Invyswell: A hybrid transactional memory for Haswell's restricted transactional memory In 2014 23rd International Conference on Parallel Architecture and Compilation Techniques (PACT). 187-199. https://doi.org/10.1145/2628071.2628086

[8] J. Chung, D. R. Chakrabarti, and C. Cao Minh. 2010. Analysis on semantic transactional memory footprint for hardware transactional memory. In Workload Characterization (IISWC), 2010 IEEE International Symposium on. 1-11. https: //doi.org/10.1109/IISWC.2010.5649529

[9] J. Chung, L. Yen, S. Diestelhorst, M. Pohlack, M. Hohmuth, D. Christie, and D. Grossman. 2010. ASF: AMD64 Extension for Lock-Free Data Structures and Transactional Memory. In 2010 43rd Annual IEEE/ACM International Symposium on Microarchitecture. 39-50. https://doi.org/10.1109/MICRO.2010.40

[10] Peter Damron, Alexandra Fedorova, Yossi Lev, Victor Luchangco, Mark Moir and Daniel Nussbaum. 2006. Hybrid Transactional Memory. In Proceedings of the 12th International Conference on Architectural Support for Programming Languages and Operating Systems (ASPLOS XII). ACM, New York, NY, USA, 336346. https://doi.org/10.1145/1168857.1168900

[11] Dave Dice, Timothy L. Harris, Alex Kogan, Yossi Lev, and Mark Moir. 2014. Pitfalls of Lazy Subscription. In Workshop on the Theory of Transactional Memory (WTTM '14).

[12] Stephan Diestelhorst, Martin Nowack, Michael Spear, and Christof Fetzer. 2013 Brief Announcement: Between All and Nothing - Versatile Aborts in Hardware Transactional Memory. In Proceedings of the Twenty-fifth Annual ACM Symposium on Parallelism in Algorithms and Architectures (SPAA '13). ACM, New York, NY, USA, 108-110. https://doi.org/10.1145/2486159.2486165

[13] Aleksandar Dragojević, Rachid Guerraoui, Anmol V. Singh, and Vasu Singh. 2009. Preventing Versus Curing: Avoiding Conflicts in Transactional Memories. In ACM Symp. on Principles of Distributed Computing (PODC '09). 7-16. https: //doi.org/10.1145/1582716.1582725

[14] Lance Hammond, Vicky Wong, Mike Chen, Brian D. Carlstrom, John D. Davis Ben Hertzberg, Manohar K. Prabhu, Honggo Wijaya, Christos Kozyrakis, and Kunle Olukotun. 2004. Transactional Memory Coherence and Consistency. In Proceedings of the 31st Annual International Symposium on Computer Architecture (ISCA '04). IEEE Computer Society, Washington, DC, USA, 102-. http://dl.acm. org/citation.cfm?id=998680.1006711

[15] Tim Harris, James Larus, and Ravi Rajwar. 2010. Transactional memory. Synthesis Lectures on Computer Architecture 5, 1 (2010), 1-263.

[16] Maurice Herlihy, Victor Luchangco, Mark Moir, and William N. Scherer, III. 2003. Software Transactional Memory for Dynamic-sized Data Structures. In Proceedings of the Twenty-second Annual Symposium on Principles of Distributed Computing (PODC '03). ACM, New York, NY, USA, 92-101. https://doi.org/10. $1145 / 872035.872048$

[17] Intel Corporation. 2012. Intel® Architecture Instruction Set Extensions Programming Reference.

[18] C. Jacobi, T. Slegel, and D. Greiner. 2012. Transactional Memory Architecture and Implementation for IBM System Z. In Microarchitecture (MICRO), 2012 45th Annual IEEE/ACM International Symposium on. 25-36. https://doi.org/10.1109/ MICRO.2012.12

[19] Sanjeev Kumar, Michael Chu, Christopher J. Hughes, Partha Kundu, and Anthony Nguyen. 2006. Hybrid Transactional Memory. In Proceedings of the Eleventh ACM SIGPLAN Symposium on Principles and Practice of Parallel Programming (PPoPP '06). ACM, New York, NY, USA, 209-220. https://doi.org/10.1145/1122971.1123003

[20] M. Lupon, G. Magklis, and A. Gonzalez. 2009. FASTM: A Log-based Hardware Transactional Memory with Fast Abort Recovery. In Parallel Architectures and Compilation Techniques, 2009. PACT '09. 18th International Conference on. 293-302. https://doi.org/10.1109/PACT.2009.19

[21] W. Maldonado, P. Marlier, P. Felber, J. Lawall, G. Muller, and E. Rivière. 2011 Deadline-aware scheduling for Software Transactional Memory. In Dependable Systems Networks (DSN), 2011 IEEE/IFIP 41st International Conference on. 257-268. https://doi.org/10.1109/DSN.2011.5958224
[22] Austen McDonald, JaeWoong Chung, Brian D. Carlstrom, Chi Cao Minh, Hassan Chafi, Christos Kozyrakis, and Kunle Olukotun. 2006. Architectural Semantics for Practical Transactional Memory. In Proceedings of the 33rd Annual International Symposium on Computer Architecture (ISCA '06). IEEE Computer Society, Washington, DC, USA, 53-65. https://doi.org/10.1109/ISCA.2006.9

[23] Chi Cao Minh, Jaewoong Chung, Christos Kozyrakis, and Kunle Olukotun. 2008. STAMP: Stanford Transactional Applications for MultiProcessing. In IEEE International Symposium on Workload Characterization. 35-46. https: //doi.org/10.1109/IISWC.2008.4636089

[24] N. Nethercote, R. Walsh, and J. Fitzhardinge. 2006. "Building Workload Characterization Tools with Valgrind". In 2006 IEEE International Symposium on Workload Characterization. 2-2. https://doi.org/10.1109/IISWC.2006.302723

[25] James Poe, Chang-Burm Cho, and Tao Li. 2008. Using Analytical Models to Efficiently Explore Hardware Transactional Memory and Multi-Core Co-Design. Computer Architecture and High Performance Computing, Symposium on 0 (2008), 159-166. https://doi.org/10.1109/SBAC-PAD.2008.18

[26] Jose Renau, Basilio Fraguela, James Tuck, Wei Liu, Milos Prvulovic, Luis Ceze, Smruti Sarangi, Paul Sack, Karin Strauss, and Pablo Montesinos. 2005. SESC simulator. http://sesc.sourceforge.net.

[27] Michael F. Spear, Arrvindh Shriraman, Luke Dalessandro, Sandhya Dwarkadas, and Michael L. Scott. 2007. Nonblocking Transactions Without Indirection Using Alert-on-update. In Proceedings of the Nineteenth Annual ACM Symposium on Parallel Algorithms and Architectures (SPAA '07). ACM, New York, NY, USA, 210-220. https://doi.org/10.1145/1248377.1248414

[28] Haris Volos, Andres Jaan Tack, Neelam Goyal, Michael M. Swift, and Adam Welc. 2009. xCalls: Safe I/O in Memory Transactions. In Proceedings of the 4th ACM European Conference on Computer Systems (EuroSys '09). ACM, New York, NY, USA, 247-260. https://doi.org/10.1145/1519065.1519093

[29] Adam Welc, Bratin Saha, and Ali-Reza Adl-Tabatabai. 2008. Irrevocable Transactions and Their Applications. In Proceedings of the Twentieth Annual Symposium on Parallelism in Algorithms and Architectures (SPAA '08). ACM, New York, NY, USA, 285-296. https://doi.org/10.1145/1378533.1378584

[30] Michael Wong and Victor Luchangco. 2014. SG5 Transactional Memory Support for $\mathrm{C}++$ Update. std.org/jtc1/sc22/wg21/docs/papers/2014/n4180.pdf

SG5 Transac-
ttp://www.open-

1] Richard M. Yoo, Christopher J. Hughes, Konrad Lai, and Ravi Rajwar. 2013. Performance Evaluation of Intel\&Reg; Transactional Synchronization Extensions for High-performance Computing. In Proceedings of the International Conference on High Performance Computing, Networking, Storage and Analysis (SC '13). ACM, New York, NY, USA, Article 19, 11 pages. https://doi.org/10.1145/2503210.2503232

[32] Craig Zilles and Ravi Rajwar. 2007. Transactional Memory and the Birthday Paradox. In Proceedings of the Nineteenth Annual ACM Symposium on Parallel Algorithms and Architectures (SPAA '07). ACM, New York, NY, USA, 303-304. https://doi.org/10.1145/1248377.1248428

[33] Ferad Zyulkyarov, Tim Harris, Osman S. Unsal, Adrían Cristal, and Mateo Valero. 2010. Debugging Programs That Use Atomic Blocks and Transactional Memory. In Proceedings of the 15th ACM SIGPLAN Symposium on Principles and Practice of Parallel Programming (PPoPP '10). ACM, New York, NY, USA, 57-66. https: //doi.org/10.1145/1693453.1693463 